\section{LAS UNIVERSIDADES JESUITAS Y LA CIENCIA DESPUÉS DE LA RESTAURACIÓN DE LA COMPAÑÍA DE JESÚS (1814-2014)}

\author{
Agustín Udías Vallina \\ Universidad Complutense de Madrid \\ audiasva@ucm.es
}

\section{JESUIT UNIVERSITIES AND SCIENCE AFTER SOCIETY OF JESUS RESTORATION OF THE (1814-2014)}

Cómo citar este artículo/Citation: Udías Vallina, A. (2016). Las universidades jesuitas y la ciencia después de la restauración de la Compañía de Jesús (1814-2014). Arbor, 192 (782): a361. doi: http://dx.doi.org/10.3989/arbor.2016.782n6006
Copyright: (c) 2016 CSIC. Este es un artículo de acceso abierto distribuido bajo los términos de la licencia Creative Commons Attribution (CC BY) España 3.0.

Recibido: 04 noviembre 2014. Aceptado: 25 agosto 2015.

RESUMEN: Los jesuitas, siguiendo la tradición desde su fundación en la labor educacional, después de su restauración en 1814, fundaron por todo el mundo una red de universidades que hoy cuenta con unas 130. La mayoría de estas universidades cuentan con departamentos de ciencias en los que jesuitas y colaboradores seglares han realizado y continúan realizando en el presente una importante labor científica. En muchas de estas universidades y en algunos colegios se crearon observatorios dedicados a astronomía, meteorología, geofísica y sismología, cuya labor científica floreció sobre todo entre 1824 y 1980. De esta forma los jesuitas continúan su presencia en distintos campos de las ciencias naturales que habían comenzado desde su fundación. Esta peculiar tradición científica de los jesuitas se explica por su propia espiritualidad ignaciana, que busca encontrar a Dios en todas las cosas, en la unión del trabajo y la oración y la preferencia por el trabajo en las fronteras. Esta espiritualidad sigue estando presente hoy detrás del trabajo científico en las universidades jesuitas, tanto en los jesuitas mismos como en sus colaboradores seglares.

PALABRAS CLAVE: Universidades jesuitas; tradición científica; observatorios; espiritualidad ignaciana.
ABSTRACT: After the restoration in 1814, the Jesuits, following an educational tradition present since their inception, established a worldwide network of around 130 universities. Many of these universities have science departments where Jesuits and their lay collaborators carry out important scientific research. A significant number of the universities and some schools set up observatories devoted to astronomy, meteorology, geophysics and seismology, where scientific work flourished, especially between 1824 and 1980. In this way, the Jesuits have constantly been present in different fields of the natural sciences since their foundation. This unusual scientific tradition can be explained by their Ignatian spirituality, which seeks to find God in all things, in the union of work and prayer and their preference for working at the frontiers. This spirituality continues today in the scientific work of Jesuit universities by Jesuits and their lay collaborators.

KEYWORDS: Jesuit universities; scientific tradition; observatories; Ignatian spirituality. 


\section{LAS NUEVAS UNIVERSIDADES JESUITAS}

Unos años después de su aprobación en 1540, la Compañía de Jesús empezó a preocuparse por el problema de la educación, empezando por la formación de sus propios miembros que habían entrado sin la formación suficiente. En 1544 tenía ya siete colegios o residencias para sus estudiantes cerca de las universidades de París, Lovaina, Colonia, Padua, Alcalá de Henares, Valencia y Coímbra en las que estudiaban. Poco más tarde, empezarían sus propias instituciones, encargándose ellos mismos de la docencia, en las que había estudiantes tanto jesuitas como seglares. En 1546 a instancias y con el apoyo de San Francisco de Borja (1510-1572), todavía duque de Gandía y que más tarde entraría en la Compañía y en 1565 sería elegido General de la Orden, se creó un colegio en Gandía, al que el papa Pablo III concedió el rango de universidad, convirtiéndose en la primera universidad jesuita, aunque su realidad fue muy modesta. En 1548 se crea en Mesina el que se considera el primer colegio jesuita que pronto se convertiría en universidad, con ya las características que tendrían después todos los colegios y universidades, con profesorado exclusivamente jesuita, alumnado seglar y rentas fijas, que permitían dar una enseñanza gratuita. Las universidades tenían facultades superiores de filosofía, teología y leyes y necesitaban una concesión por el papa o reyes para conceder grados académicos y otros privilegios. Para San Ignacio de Loyola (1491-1556), la creación de colegios y universidades constituyó una orientación nueva de la orden recién fundada, con la que no había contado al principio, y la enseñanza se convirtió, ya durante su vida, en el instrumento más importante de su trabajo apostólico. A su muerte en 1556 la Compañía tenía 35 colegios y ocho universidades en diversos países de Europa. El Colegio Romano, fundado en 1551, recibió en 1556 del papa Pablo IV el rango de universidad con docencia de filosofía y teología, que sería confirmado por Pablo V en 1576. En 1584 se inaugura el magnífico nuevo edificio con el patronazgo de Gregorio XIII, y desde 1873 lleva el nombre de Universidad Gregoriana, en recuerdo de su patrono. Esta institución, a la que el mismo San Ignacio dio una gran importancia, se convirtió en el modelo a seguir por los demás colegios y universidades jesuitas (García Villoslada, 1954). De esta forma pocos años después de su fundación la Compañía tomaba la labor de la enseñanza como el elemento clave de su labor apostólica. La rápida extensión de los colegios y universidades jesuitas se explica por la necesidad y demanda social de la educación de la juventud, a la que la nueva orden respondió con un nuevo estilo y estructura pedagógica. Por otro lado, este método de apostolado casaba bien con una orden originalmente formada por diez Maestros de Artes de la Universidad de París.

Con la restauración de la Compañía en 1814, después de su supresión por el papa Clemente XIV en 1773, los jesuitas se encontraron con una situación nueva respecto, tanto a la enseñanza en general como a la ciencia durante el siglo XIX. Esto va a afectar a la creación de los nuevos colegios y universidades. En general, la enseñanza quedaba dividida en tres niveles: primaria, media o secundaria y superior o universitaria. En la mayoría de los países, se establece su dependencia del estado a los tres niveles y se considera la educación como un servicio público. De esta forma es prerrogativa de los gobiernos el establecer la reglamentación y planes de estudio que deben seguirse a los tres niveles. En cuanto a los contenidos de los niveles medios y superior se establece un definido énfasis en los estudios de ciencias. Las universidades, que habían sufrido una general decadencia en Europa durante el siglo XVIII, conocen en el siglo XIX un proceso de reforma, en el que se pone el énfasis en el estudio de las ciencias naturales. Las universidades alemanas, empezando con las de Berlín y Gotinga, se destacaron además por ser pioneras en dar un papel predominante a la investigación. Esta nueva doble orientación, docenciainvestigación, que se extenderá a todas las universidades modernas, supone un cambio radical respecto a la universidad heredada de la Edad Media centrada en la docencia. En Francia, durante el periodo napoleónico, se llevó también a cabo un proceso profundo de reforma y modernización de todas las universidades, que pasaron a depender directamente del estado. Este proceso de estatalización de las universidades se extendió a otros países europeos como Italia y España. La teología, que había ocupado un puesto predominante durante la Edad Media, había ido perdiendo su importancia en el campo académico y en muchos casos desaparece su docencia, cediendo en las universidades su puesto al estudio de las ciencias naturales.

La supresión de la Compañía había supuesto la pérdida de las universidades que había regentado durante los siglos XVI al XVIII, en Europa y América. Cuando se restaura en 1814, la situación respecto a las universidades había cambiado muy radicalmente, como hemos visto, y su estatalización hacía prácticamente imposible a los jesuitas el recuperar las instituciones universitarias en Europa, con excepción de las destinadas a estudios eclesiásticos. En otros países la situación era distinta, como en Estados Unidos, donde la Compañía pudo empezar a fundar instituciones de enseñanza superior a partir de 
mediados del siglo XIX. Otro ejemplo es el de la India, donde siguiendo el modelo inglés, los jesuitas pudieron regentar colleges asociados a universidades estatales con enseñanza a nivel superior. Con el tiempo la situación ha ido cambiando y los jesuitas han podido fundar universidades en otros países incluidos los europeos.

Actualmente existen unas 130 universidades jesuitas en el mundo, fundadas las primeras entre mediados del siglo XIX y principios del XX. Incluidos otros centros de enseñanza superior suman 231 en más de 70 países, con más de 18.000 miembros jesuitas y más de 160.000 profesores laicos. En Estados Unidos, donde existía una tradición de instituciones universitarias privadas, los jesuitas pudieron, poco después de su restauración, establecer las primeras universidades. La primera de ellas fue la Universidad de Georgetown en Washington D.C., que había sido creada en 1789 , durante la supresión, por John Carroll (1738-1815), exjesuita, primer obispo católico de Baltimore. Después de la restauración la universidad pasó a depender de la Compañía. De las 28 universidades actualmente existentes, 19 se fundaron antes de 1900. Las que se pueden considerar como las más importantes son: Georgetown (1789), Saint Louis, Missouri (1818), Fordham, Nueva York (1841), Boston College, Massachusetts (1863), Loyola, Chicago (1870), San Francisco, California (1875), Regis, Denver (1877), Marquette, Milwaukee (1881). Las universidades jesuitas en Estados Unidos experimentaron un rápido crecimiento entre los años 1950 y 1960, tanto en alumnado como en profesorado y recursos y su expansión ha continuado hasta el presente (Schroth, 2007, pp. 174-196; McKevitt, 2008). En la India, los colleges jesuitas son 16 en la actualidad. Entre ellos destacan los siguientes: Xavier's, Kolkata (1860), St. Xavier's, Mumbay (1869) y Loyola, Chennai (1925). En Europa hay ocho universidades jesuitas, tres de ellas en España. En América Central y del Sur los jesuitas regentan 24 universidades. Entre ellas destacan: Pontificia Universidad Javeriana, Bogotá $(1623,1937)$, Pontificia Universidade Catolica de Rio de Janeiro, Brasil (1940) y Universidad Iberoamericana, México (1943). En Asia, además de los colleges ya mencionados de la India, hay 11 universidades entre ellas: Ateneo de Manila University, Filipinas (1859), Sofia University, Tokio (1913), Sogang University, Seúl (1960). En África y Oriente Próximo hay cinco universidades jesuitas.

\section{LA CIENCIA EN LAS UNIVERSIDADES JESUITAS}

En las nuevas universidades jesuitas, siguiendo la tradición científica de las instituciones de la antigua Compañía, se dio un papel importante a las ciencias
(Udías, 2014, pp. 295-308). De esta forma, en la mayoría hay programas de ciencias, aunque varían mucho los campos científicos presentes y la calidad de los departamentos científicos, tanto en docencia como en investigación. Por otro lado, la presencia misma de profesores jesuitas en los departamentos de ciencias de las distintas universidades varía mucho, también, de unas a otras y con el tiempo. Originalmente, la presencia de jesuitas entre el profesorado de ciencias era relativamente alta y ha disminuido mucho recientemente, de forma que actualmente el profesorado está formado mayoritariamente por seglares. En Estados Unidos y en la India, donde existe el mayor número de colleges y universidades con departamentos de ciencias, es donde se puede encontrar el mayor número de profesores jesuitas en los distintos campos de la ciencia, aunque ha disminuido notablemente en los últimos años. Aunque un cierto nivel de investigación ha estado siempre y sigue estando presente, se puede decir que su principal dedicación es, en la mayoría de los casos, la docencia. Al principio de su fundación, en las universidades que poseían observatorios, se llevó a cabo investigación en los campos de astronomía, geofísica y meteorología. Las ciencias naturales, sobre todo botánica y entomología, conocieron también la presencia de importantes investigadores jesuitas, como veremos más adelante. En Europa hay pocas instituciones de enseñanza superior con programas de ciencias. En Bélgica hay departamentos de ciencias en la Universidad de Namur, fundada en 1831 con el nombre de las Facultés Universitaires Notre Dame de la Paix. Con carácter más tecnológico está en Francia el Institut Catholique d'Arts et Métiers (ICAM), Lille, fundado en 1898 y que se amplió en 1911 a estudios superiores de ingeniería. En España las instituciones jesuitas de enseñanza superior de carácter científico y técnico empezaron a principios del siglo XX. En relación con la Facultad de Filosofía en Roquetas (Tarragona) se proyectó la creación de tres laboratorios de física, química y biología. El primero se cambió a un observatorio dedicado a las relaciones entre el magnetismo terrestre y la actividad solar que se fundó en 1904 con el nombre de Observatorio del Ebro. En 1905 se creó el Laboratorio Químico del Ebro, y en 1908 el Laboratorio Biológico del Ebro. Ambos laboratorios se trasladaron a Barcelona en 1916, cambiando el nombre a Instituto Químico de Sarriá e Instituto Biológico de Sarriá, que terminaron formando un único instituto con el nombre de Instituto Químico de Sarriá (IQS). En 1990 el IQS, junto con otras instituciones, se integraron en la Universidad Ramón Llull, universidad privada de inspiración cristiana. En Madrid, se fundó en 1908 
el Instituto Católico de Artes e Industrias (ICAI), una escuela de ingenieros inspirada en el ICAM de Lille. En 1978 el ICAI se integró en la institución jesuita Universidad Pontificia Comillas. En la Universidad de Deusto, fundada en 1886, existen actualmente varias ramas de estudios de ingeniería. La recientemente fundada (2013) Universidad Loyola Andalucía tiene una rama de ingeniería.

\section{LOS OBSERVATORIOS}

Uno de los primeros campos científicos en el que destacaron las universidades jesuitas fue la astronomía con la creación de observatorios (Udías, 2003). El primero de los nuevos observatorios fue el del Colegio Romano que se llamaría más tarde Universidad Gregoriana. Pietro Angelo Secchi (1818-1878), fue nombrado director del observatorio en 1850. Secchi es uno de los grandes jesuitas científicos de esta nueva época, destacando por sus estudios astrofísicos de la espectroscopía del sol y las estrellas, en especial su clasificación de los espectros de las estrellas, base de la usada actualmente (Altamore y Maffeo, 2012). La labor del observatorio no duró mucho pues en 1870 los jesuitas fueron expulsados y el Colegio Romano con el observatorio confiscado por el gobierno italiano. Sin embargo, a Secchi, debido a su prestigio internacional se le permitió continuar como director hasta su muerte, ocho años más tarde. Asociados a colegios de segunda enseñanza fueron los observatorios de Stonyhurst en Inglaterra fundado en 1838 en el que destacó Stephen Perry (1833-1889), uno de los cuatro jesuitas miembros de la prestigiosa Royal Society de Londres (Secchi fue otro) y director de varias expediciones científicas de carácter astronómico. Otro observatorio europeo importante fue el de Kalocsa en Hungría, donde Gyula Fenyi (1845-1927) realizó numerosos estudios sobre la naturaleza de la actividad del sol.

El observatorio astronómico más importante instalado por los jesuitas en Norteamérica fue el de la Universidad de Georgetown. El observatorio se inauguró en 1844 en un edificio construido con este motivo con una gran cúpula en la que además de otros instrumentos se colocó en 1849 un telescopio de montura ecuatorial de $12 \mathrm{~cm}$ de apertura. El observatorio fue el cuarto establecido en los Estados Unidos, solo seis años después del primero, Hopkins Observatory en Williamstown. Entre 1888 y 1906 estuvo de director Johann Georg Hagen (1847-1930) que destacó por sus investigaciones sobre las estrellas variables. En 1899 Hagen empezó la publicación de su gran obra Atlas stellarum variabilium (Atlas de estrellas variables). En
1906 fue nombrado el primer director jesuita del Observatorio Vaticano. De 1927 a 1948 fue director Paul A. McNally (1890-1955) que participó en varias expediciones para observar eclipses del Sol, por ejemplo, en 1936 en Siberia y en 1937 en el sur del Pacífico. Le sucedió Francis J. Heyden (1907-1991), que creó un programa de estudios graduados de astronomía en la universidad. Con la colaboración de sus estudiantes lanzó una serie de proyectos de investigación en espectrografía solar, radio astronomía y el estudio de atmosferas planetarias. Heyden participó también en expediciones para observar eclipses de sol en 1947 en Brasil y 1948 en China. Otros observatorios astronómicos menores se instalaron entre 1880 y 1909 en las universidades jesuitas de Creighton (Omaha), Santa Clara (California) y Marquette (Milwaukee).

En la India en 1866 en el prestigioso St. Xavier College de Calcuta (Kolkata), se instaló una estación meteorológica para fomentar la enseñanza de las ciencias y en 1874 se instaló una cúpula y un telescopio ecuatorial Merz de $18 \mathrm{~cm}$ de apertura con otros instrumentos astronómicos, en el que destacó la labor del jesuita Eugene Lafont (1837-1908), profesor de ciencias. Lafont fue una figura importante en el desarrollo de los estudios de ciencias en la India. En Méjico los jesuitas fundaron dos observatorios en los colegios de Puebla en 1877 y de Saltillo en 1884. El del Colegio de Puebla que poseía secciones de astronomía, meteorología, magnetismo y sismología fue fundado por el jesuita italiano Pedro Spina (1839-1925). Este fue el segundo observatorio astronómico instalado en Méjico dos años más tarde que el Observatorio Nacional de Chapultepec. La Facultad de Filosofía y Teología en San Miguel, cerca de Buenos Aires, Argentina, instaló en 1935 el Observatorio de Física Cósmica dedicado a astrofísica, rayos cósmicos, actividad solar y fenómenos electromagnéticos. En él destacó Juan Antonio Bussolini (1905-1966), quien dio un gran impulso a los trabajos científicos.

En los observatorios instalados en universidades jesuitas se añadieron pronto a la astronomía las observaciones meteorológicas, sismológicas y del campo magnético terrestre. Sismología se convirtió pronto un campo de especial interés de los jesuitas científicos (Udías y Stauder, 1996). En 1911 en Estados Unidos quince universidades jesuitas formaron una red de estaciones sismológicas con instrumentación uniforme, la primera red de estas características en el país y única en el mundo cubriendo todo un continente. En 1925 la red fue renovada con el nombre de Jesuit Seismological Association por James Macelwane (1883-1956), 
profesor de la Universidad de Saint Louis (Missouri) y el único jesuita miembro de la American National Academy of Science. La red funcionó hasta 1970, cuando los servicios que ofrecía empezaron a ser cubiertos por instituciones estales (Macelwane, 1950). Macelwane fue pionero en los estudios de la estructura del interior de la tierra por métodos sismológicos. Daniel Linehan (1904-1987) fue director del Weston Seismological Observatory, que formaba parte de la universidad jesuita, Boston College (Massachusetts). Creó en 1948 el Departamento de Geofísica, en el que se formaron varios jesuitas que luego trabajaron en otros observatorios. Linehan amplió el Observatorio con una red sísmica en el nordeste de Estados Unidos, que entre 1955 y 1980, se amplió de 6 a 30 estaciones. El departamento de Geofísica de la Universidad de Saint Louis y el Observatorio de Weston continúan hoy como centros prestigiosos de investigación sismológica. Varios observatorios sismológicos fueron dirigidos por jesuitas en América Central y del Sur, pero solo el Instituto Geofísico en Bogotá está asociado a una universidad, la Universidad Javeriana. Su fundador fue Emilio Ramírez (1904-1981), también durante un tiempo rector de la universidad, que destacó por sus estudios de sismología y tectónica de América del Sur.

La meteorología fue otra ciencia a la que contribuyeron colegios y universidades jesuitas (Udías, 1996). Fueron muchos los colegios y universidades jesuitas en las se instalaron estaciones meteorológicas, que contribuyeron al desarrollo de esta ciencia desde los finales del siglo XIX. Entre las universidades con programas de meteorología destacan el Colegio Romano (Universidad Gregoriana), la Universidad de Saint Louis, única que mantiene hoy un programa graduado en esta ciencia, la Universidad de John Carrol (Cleveland), Santa Clara, Spring-Hill College y la Universidad Javeriana (Bogotá). El estudio de los ciclones tropicales fue un área en cuyo estudio destacaron científicos jesuitas. En el Real Colegio de Belén en la Habana, creado en 1854, se instaló un observatorio meteorológico, que llevaría el nombre de Observatorio de Belén, y que se haría famoso por sus estudios de los ciclones tropicales desde 1870, bajo la dirección de Benito Viñes (1837-1893). Sobre la base de las observaciones meteorológicas desde su fundación, Viñes estudió con detalle las características de los ciclones que habían afectado la isla de Cuba, acumulando conocimientos sobre su organización, circulación y traslación. Recogió los resultados de sus investigaciones en su pionero libro Investigaciones relativas a la circulación y traslación ciclónica de los huracanes de las Antillas (1895). En Filipinas, en el Ateneo de Manila, hoy Universidad, se estableció un observatorio meteorológico, sismológico y astronómico en 1865, que destacó por los estudios de los tifones con los trabajos de Federico Faura (1840-1897), José Algué (18561930) y Charles Deppermann (1889-1957). En China, el observatorio de Zikawei en Shanghai, fundado en 1872, que destacó con los estudios y seguimiento de los ciclones del mar de China, estaba asociado a la Universidad Aurora desde su fundación por los jesuitas en 1903. En él destacaron por sus estudios sobre la naturaleza de los ciclones y el seguimiento de sus trayectorias Marc Dechevrens (1845-1923), Louis Froc (1859-1932) y Ernesto Gherzi (1886-1976).

\section{OTROS CAMPOS CIENTÍFICOS}

Otro campo científico importante en que destacaron algunas universidades jesuitas fue el de la biología. El fundador del Instituto Biológico de Sarriá (Barcelona), Jaime Pujiula (1869-1958) fue un pionero de la investigación embriológica, histológica y citológica en España. Entre sus libros se encuentran Citología (1928) e Histología, embriología y anatomía humana y animal (1934). En los colleges establecidos por los jesuitas en la India existe una brillante tradición de estudios de botánica con aplicaciones a la agricultura, el medio ambiente y la ecología que se continúan en nuestros días (D'Souza, 2009). Entre los primeros figura el suizo Ethelbert Blatter (1877-1934) que llegó a la India en 1903 y fue profesor de St. Xavier College, Mumbai y miembro de la Bombay Natural History Society. En el colegio estableció el Blatter Herbarium formado por cerca de tres millones de plantas. Entre sus libros se encuentran The palms of British India and Ceylan (1926). En St. Xavier College fue también profesor Hermenegildo Santapau (1903-1970), originario de Tarragona, doctor por la Universidad de Londres, que desarrolló un extenso trabajo de botánica. Fue presidente en 1955 de la Indian Botanical Society y director entre 1961 y 1967 del Botanical Survey de la India. Publicó 216 artículos y monografías sobre la flora de diversas partes de la India, entre sus libros se encuentra The Flora of Purandhar (1958). Koyapillil M. Mathew (1930-2004), nacido en Kerala y profesor de botánica en St. Joseph College, Tiruchirappalli, dedicó sus esfuerzos al estudio y conservación de los bosques shola de la región subtropical del sur de la India. Eminente taxonomista entre sus libros se cuenta, The flora of the Palni Hills (1999). Mathew organizó el Rapinat Herbarium, colección de más de 54000 plantas recogidas y catalogadas por los jesuitas desde 1895, y fomentó el aprecio de la población de la región por sus propias plantas. Cecil Saldanha (1926-2002), pro- 
fesor de botánica en St. Joseph's College, Bangalore, dedicó sus esfuerzos al estudio de la flora y el medio ambiente del Estado de Karnataka. Entre sus publicaciones, Flora of Karnataka (2 vols. 1978), donde se describen 1700 especies. Los estudios de botánica, botánica aplicada, medio ambiente y ecología están hoy presentes en muchos de los colleges jesuitas, en especial, los de St. Xavier's (Mumbai), St. Xavier's (Ahmedabad), Loyola (Chennai), St. Joseph's (Tiruchiparapalli), St. Aloysius (Mangalore), St. Xavier's (Kolkatta) y St. Xavier (Palayamkottai).

Dos entomólogos españoles fueron profesores de colegios de segunda enseñanza y merecen citarse aquí. Pelegrín Franganillo (1873-1955) comenzó sus estudios de las arañas en Galicia y Asturias que publicó en Las arañas. Manual de aracnología (1917). En 1918 se trasladó a Cuba, al Colegio de Belén en la Habana, donde continuó sus estudios sobre las arañas (Arácnidos de Cuba, 1923). Profesor del Colegio de Zaragoza, Longinos Navás (1858-1938), destacó por sus estudios sobre neurópteros de los que llegó a clasificar 1786. En total clasificó un total de 3316 formas nuevas (388 géneros, 2684 especies y 244 variedades). Trabajador infatigable, entre 1898 y 1935, publicó 588 artículos en revistas científicas y actas de congresos. Entre sus monografías científicas destaca Entomologia de Catalunya. Neuròpters (fascicles 1-3, 1923, 1926, 1934).

Algunos jesuitas destacaron como eminentes profesores de ciencias, por ejemplo, en España Eduardo Vitoria (1864-1958), profesor del IQS, publicó en 1910, Manual de química moderna, con 15 ediciones, la última en 1955. José Agustín Pérez del Pulgar (18751939) destacó por sus estudios de electricidad y física matemática y publicó en cuatro volúmenes Electrodinámica Industrial (1915-1919). Entre los profesores jesuitas de ICAI destaca Enrique de Rafael (18851955) que entró en la Compañía después de obtener su doctorado en Matemáticas y ser profesor auxiliar en la Universidad de Barcelona. Entre 1922 y 1933 enseñó matemáticas y astronomía en St. Xavier's College, Mumbai y en 1939 se incorporó al ICAI. En 1943 ingresó en la Real Academia de Ciencias.

\section{LOS LIBROS DE TEXTO}

Siguiendo la tradición de los científicos de la antigua Compañía se dio también entre los profesores de las nuevas universidades la composición de libros de texto de ciencias. Algunos ejemplos son: Angelo Secchi (Collegio Romano), Lezioni di Fisica Terrestri (1877); Theodore Wulf (Valkenburgkollege, Holanda), Lehrbuch der Physick (1926); James Macelwane (Saint
Louis University), Introduction to theoretical seismology (1936); August Bellynck y Egide Pâque (Universidad de Namur), Cours de Botanique (1899), Joseph Bayma (Santa Clara University, California), Elements of Infinitesimal Calculus (1889), Adolf Müller (Universidad Gregoriana, Roma) Elementi di astronomía (1904); Eugenio Saz, (IQS, Barcelona) Fundamentos de química general (1924), Edmund J. Burke (Fordham University, Nueva York), Lectures in biology: zoology (1928); Claudio Mataix (ICAI, Madrid), Mecánica de fluidos y máquinas hidráulicas (1970).

\section{EL TRABAJO CIENTÍFICO Y LA ESPIRITUALIDAD DE LOS JESUITAS}

La Compañía restaurada, a pesar de las dificultades que encontró al principio, creó un número considerable de universidades en las que las ciencias han ocupado y ocupan hoy un lugar importante. Por ejemplo, como hemos visto los jesuitas dirigieron más de 70 observatorios astronómicos y geofísicos, que florecieron entre finales del siglo XIX y mediados del XX, muchos de ellos vinculados a universidades. Queda por preguntarnos qué hay de especial en los jesuitas que explique esta dedicación continuada a las ciencias tanto en las universidades como fuera de ellas (Udías, 2012). No cabe duda que otras órdenes y congregaciones religiosas han tenido personalidades científicas de relieve, por ejemplo, Gregor Mendel, pionero de la genética, de la orden de San Agustín y Georges Lemaitre, primero en proponer la idea del big-bang, sacerdote secular. Sin embargo, en el caso de los jesuitas no se trata de casos particulares, sino de una presencia continuada e institucional, desde los principios de su fundación hasta el presente, como hemos visto en el caso de las universidades creadas después de la restauración en 1814. Por ejemplo, es indicativo de esta presencia el hecho que en 1906, 18 años después de su fundación, Pio X encargara a los jesuitas la dirección del Observatorio Vaticano, que se mantiene en el presente.

Podemos buscar algunos motivos de esta dedicación en concreto en la época moderna. Además de la tradicional dedicación a la ciencia, ya presente en la Compañía antigua, en esta época la presencia de los jesuitas en las ciencias se justificaba como un argumento apologético en contra de los que atacaban a la Iglesia como enemiga de la ciencia. Esta actitud se había extendido y generalizado sobre todo a partir de finales del siglo XIX, con la propagación de la opinión de una total incompatibilidad entre ciencia y religión, que sigue aún viva en algunos ambientes. La presencia de jesuitas en ciencias y la existencia de sus propias 
instituciones científicas se presentaban como un claro argumento contra estas falsas acusaciones, y un ejemplo de la compatibilidad entre fe cristiana y ciencia. Aloysius Cortie (1859-1925), director del Observatorio de Stonyhurst, escribiendo en 1923 sobre Secchi, decía: "Los enemigos de la Santa Iglesia han hecho un injustificado uso de la ciencia como un arma de ataque contra sus más fundamentales verdades que se ha creado una impresión entre muchos de sus hijos de que el cultivo de la ciencia es perjudicial y peligroso para la fe....el P. Secchi es un ejemplo admirable de quien supo unir religión y ciencia" (Cortie, 1923).

Además de estos factores de tipo histórico, vinculados a las necesidades apostólicas relacionadas con la enseñanza en los colegios y universidades, hay que buscar también elementos más específicos de los jesuitas. Uno de los primeros estudios que tratan de dar una respuesta a esta pregunta es el de Steven J. Harris (Harris, 1989). Aunque su análisis está basado solo en los jesuitas de los siglos XVII y XVIII, sus ideas se pueden aplicar también a los de la Compañía moderna y en concreto al trabajo en las universidades. Harris pone el fundamento de la dedicación de los jesuitas a la ciencia en lo que él llama su "espiritualidad apostólica", que está en el núcleo de la "ideología jesuita". El término ideología jesuita lo usó por primera vez Rivka Feldhay para referirse a la consideración del conocimiento como camino de salvación, que ella encontraba como una nota peculiar del trabajo de los jesuitas, en especial el académico (Feldhay, 1987). Para Harris los principales elementos de la espiritualidad jesuita son en primer lugar el énfasis puesto en el servicio que canaliza los sentimientos religiosos "hacia fuera", incluyendo actividades seculares, que no se asocian generalmente con la vida religiosa, y el encuentro y compromiso con el mundo, que lleva a la comprobación por la experiencia de las ideas y proyectos. En el campo científico de esta actitud se sigue la importancia dada a la observación y el experimento, es decir, a los aspectos experimentales de la ciencia. Para $\mathrm{Ha}-$ rris, la espiritualidad apostólica es la fuerza básica que conforma el carácter de la actividad de todo jesuita y está presente en su trabajo científico. Otro elemento importante, según este autor, es una estima por el conocimiento y la educación que llevó a la Compañía desde sus orígenes a la fundación de colegios y universidades y que continúa en la labor educacional actual en especial en las universidades. Finalmente está presente también lo que Harris llama una "santificación del saber", que implica compromisos con actividades seculares puestas al servicio de fines espirituales, es decir, la salvación de las almas, último fin de todo trabajo de la Compañía. Este trabajo lo ve Harris para la Compañía antigua realizado en tres ámbitos, el educacional de colegios y universidades, en las cortes europeas con las personas influyentes y en el apostolado de las misiones en lejanas tierras no cristianas.

Algunos elementos de la espiritualidad apostólica, de la que habla Harris, se pueden encontrar también en otras congregaciones religiosas modernas de carácter apostólico que, sin embargo, no han tenido una presencia similar en las ciencias. Tenemos, por lo tanto, que buscar elementos más específicos de la espiritualidad jesuita para explicar su peculiar tradición científica. Esta espiritualidad está basada en las dos grandes obras de San Ignacio, los Ejercicios espirituales y las Constituciones. En ellas se encuentran las líneas fundamentales basadas en las experiencias ascéticas y místicas del mismo San Ignacio de lo que se conoce como la "espiritualidad ignaciana" (Dhôtel, 1991). Los Ejercicios Espirituales, realizados durante un mes, dos veces, al principio y la final de la formación de todo jesuita y repetidos durante ocho días todos los años, forman el núcleo de su espiritualidad. Los Ejercicios Espirituales forman además el principal medio de la transmisión a los colaboradores seglares en las universidades de la espiritualidad ignaciana. Esto es hoy muy importante, cuando la labor científica en las universidades jesuitas está realizada en gran parte por seglares. Una de las características más importantes de la espiritualidad ignaciana es la de ser una mística del servicio, entendiendo por ello el servicio a Dios a través del ministerio apostólico con los hombres. Así está establecido en las Constituciones, al definir el fin de la Compañía de "procurar de ayudar a la salvación y perfección de las (almas) de los prójimos". Este servicio tiene además una vertiente como servicio en la Iglesia. La frase que aparece a menudo en los escritos de San Ignacio y que se ha convertido como en la divisa de los jesuitas es "para mayor gloria de Dios" (Ad maiorem Dei Gloriam), para indicar el fin último de todo trabajo del jesuita. La frase está ya en San Pablo, "hacedlo todo para Gloria de Dios" (1Cor 10,31) a la que San Ignacio añadió el comparativo "mayor", indicando la búsqueda que todo jesuita debe hacer de aquello que "más" conduce al fin buscado en cada caso, como se expresa en la meditación del "Principio y fundamento" de los Ejercicios. El "más" (en latín magis) es también una peculiaridad de esta espiritualidad. En cada momento histórico el jesuita se ve motivado por esta espiritualidad a buscar ese "más" en todos sus trabajos. Muchos jesuitas encontraron ese "más" en el trabajo científico. También tratan de comunicar esta actitud entre sus colaboradores en el trabajo científico. 
Por otro lado, insiste San Ignacio en sus escritos en "encontrar a Dios en todas las cosas", indicando una actitud de oración en la vida activa. En este aspecto es importante la última meditación de los Ejercicios Espirituales, la "Contemplación para alcanzar amor". En ella se pide al que hace los Ejercicios contemplar cómo "Dios habita en las criaturas" y cómo "trabaja y labora por mí en todas cosas criadas". Esto convierte todas las cosas, personas y circunstancias en ocasiones para encontrar a Dios. Al resaltar, también, San Ignacio la humanidad de Cristo está invitando a que todo lo humano, entre ello podemos incluir la ciencia, sea lugar de encuentro con Dios. A esta actitud se refería Jerónimo Nadal, estrecho colaborador de San Ignacio, con la expresión de ser "contemplativos en la acción", lo que implica una unión entre oración y acción; a Dios se le encuentra tanto en el silencio de la oración como en la actividad del trabajo. No hay, por lo tanto, actividad, por profana que parezca, que no pueda convertirse en oración. Enseñar matemáticas o física en una universidad, observar la luz de una galaxia lejana en un observatorio o levantar mapas de un territorio desconocido, son actividades que un jesuita encuentra perfectamente compatibles con su vocación y a través de ellas trata de encontrar a Dios en su vida. En otros grupos religiosos esta puede ser también una actitud personal en algunos de ellos, pero en el caso de los jesuitas se sigue del núcleo mismo de su espiritualidad. Así lo reconocía el astrofísico Angelo Secchi: "La contemplación de las obras de Dios es una de la nobles obras del espíritu, este es el fin principal del estudio de la naturaleza" (Secchi, 1875, p. 6). De esta manera, los jesuitas, desde el principio, se vieron inmersos en actividades, entre ellas las científicas, que otros consideraban incompatibles o no convenientes en la vida religiosa. Por eso, se puede encontrar a jesuitas dedicados al trabajo social entre marginados, ejerciendo de párrocos, investigando en biología o astrofísica, o escribiendo poesía, movidos todos por una misma espiritualidad que busca encontrar a Dios en todas las cosas. Esta espiritualidad es, además, comunicada a otros por los jesuitas en los ambientes en que se mueven y en especial entre sus colaboradores en las universidades.

Otro aspecto de la espiritualidad ignaciana en su aspecto apostólico es el de su preferencia por adentrarse en situaciones y temas de "frontera". En lugar de dedicar sus esfuerzos en actividades pastorales tradicionales, podemos decir hacia "los de dentro", los jesuitas se esfuerzan en entrar en contacto con "los de fuera", en actividades, a veces, no convencionales. Esto está reconocido en la alocución del papa Benedicto XVI a la Congregación General 35 de los jesuitas: "la Iglesia os necesita cuenta con vosotros y en vosotros sigue confiando, particularmente para alcanzar aquellos lugares físicos y espirituales a los que otros no llegan o encuentran difícil hacerlo" y se refiere a las fronteras, entre ellas "las que se alzan entre la fe y la ciencia moderna” (p. 264). Los jesuitas están siempre impulsados hacia las fronteras, hacia aquellos lugares y situaciones en los que el mensaje cristiano no es conocido. Por ejemplo, esto llevó a Ricci y sus compañeros en el siglo XVII a presentarse ante la corte imperial de China, adoptando el traje y las costumbres de los intelectuales chinos, como astrónomos de occidente y dirigir desde 1644 el Observatorio Astronómico Imperial de Beijing.

La ciencia durante el siglo XIX y principios del XX se consideraba por algunos como un campo ajeno si no hostil a la religión. Los jesuitas sintieron la responsabilidad de mostrar con su trabajo científico que no había incompatibilidad entre la ciencia y la fe, y que estos campos aparentemente profanos podían ser también un vehículo para encontrar a Dios. Esta mentalidad influyó en la creación de los departamentos de ciencias en las universidades y de los nuevos observatorios, como ya hemos visto. El jesuita científico moderno considera la comunidad científica alejada de Dios como su parroquia, rompiendo con su presencia muchas barreras de incomprensión y alejamiento. Reconociendo la unidad de su función como científico y sacerdote, Daniel Linehan, el incansable sismólogo y explorador jesuita de Boston College, grabó en la base de su cáliz: "Primera Misa en el Polo Norte Magnético, 1954" y "Primera Misa en el Polo Sur, 1958". Tenía también claras su prioridades cuando en 1956 dijo a una periodista de la revista Life: "Yo daría toda mi sismología por celebrar una Misa como a la que asististe esta mañana" (Harvey, 2000). De una u otra forma, esta actitud ha estado y está presente en los jesuitas científicos, que han tratado, también, de comunicarla a sus colaboradores en las universidades. El centro de esta espiritualidad, como ya hemos visto, está en encontrar a Dios en todas las cosas, en la unión del trabajo y la oración y la preferencia por el trabajo en las fronteras. Esta espiritualidad sigue estando presente hoy detrás del trabajo científico en las universidades jesuitas, tanto en los jesuitas mismos, como en sus colaboradores seglares, que de una forma u otra participan de ella. Para concluir, las universidades jesuitas continúan hoy la larga tradición científica, que empezaron ya en tiempos del mismo San Ignacio, a pesar de las dificultades y problemas con las que se siguen encontrando. 


\section{BIBLIOGRAFÍA}

Altamore, A. y Maffeo, S. (eds.) (2012). Angelo Secchi. L'avventura scientifica del Collegio Romano. Foligno: Quater.

Cortie, A. L. (1923). Angelo Secchi, S. J. (1818-1878). En Windle, B. (ed.). Twelve catholic men of science. Londres: Catholic Truth Society, pp. 149-164.

Dhôtel, J. C. (1991). La espiritualidad Ignaciana. Santander: Sal Terrae.

D'Souza, L. (2009). Jesuit Contribution to Environmental Protection. [En línea]. Disponible en http://www.sjweb.info/ sjs/networks/ecology/Indian\%20Jesuits $\% 20$ Contribution $\% 20$ to\%20Environmental\%20Protection.pdf

Feldhay, R. (1987). Knowledge and salvation in Jesuit culture. Science in Context, 1 , pp. 195-213. https://doi.org/10.1017/ S0269889700000363

García Villoslada, R. (1954). Storia del Collegio Romano dal suo inizio (1551) alla soppressione della Compagnia di Gesù (1773). Roma: Universidad Gregoriana.

Harris, S. J. (1989). Transposing the Merton's Thesis: Apostolic Spirituali- ty and the Establishment of the Jesuit Scientific Tradition. Science in Context, 3, pp. 29-65. https://doi.org/10.1017/ S0269889700000715

Harvey, C. B. (2000). The voyage of the Monte Carlo. Boston College Magazine. [En línea]. Disponible en http://bcm.bc.edu/ issues/fall_2000/ft_linehan.htm

Macelwane, J. B. (1950). The Jesuit Seismological Association, 1925-1950. En Macelwane, J. B. Twenty-fifth Anniversary Commemorative Volume. Jesuit Seismological Association. 1925-1950. St. Louis: Saint Louis University, pp. 17-23.

McKevitt, G. (2008). Jesuits schools in the USA, 1814-c.1970. En T. Worcester (ed.). The Cambridge Companion to the Jesuits. Cambridge: Cambridge University Press, pp. 278-293. https://doi. org/10.1017/CCOL9780521857314.017

Schroth, R. A. (2007). The American Jesuits. A history. New York: New York University Press.

Secchi, A. (1875). Le soleil. Paris: GauthierVillar
Udías, A. (1996). Jesuits' Contribution to Meteorology. Bulletin of the American Meteorological Society, 77, pp. 2307-2315. http://journals. ametsoc.org/doi/abs/10.1175/15200477\%281996\%29077\%3C2307\%3AJCT M\%3E2.0.CO\%3B2

Udías, A. (2003). Searching the Heavens and the Earth: The History of Jesuit Observatories. Dordrecht: Kluwert. https://doi. org/10.1007/978-94-017-0349-9

Udías, A. (2012). Jesuit Scientific Tradition and Ignatian Spirituality. Lo Sguardo. Rivista di Filosofia, 10, pp. 207-219.

Udías, A. (2014). Los jesuitas y la ciencia. Una tradición en la Iglesia. Bilbao: Mensajero.

Udías, A. y Stauder, W. (1996). The Jesuit Contribution to Seismology. Seismological Research Letters, 67, pp. 10-19. https://doi.org/10.1785/gssrl.67.3.10

\section{Otras fuentes}

Compañía de Jesús (2008). Congregación General 35. Bilbao: Mensajero. 